\title{
Beam Transfer at E0: An Overview
}

\author{
S. Saritepe and G. Annala \\ Fermi National Accelerator Laboratory \\ P.O. Box 500, Batavia, Illinois 60510
}

June 1993 


\section{Disclaimer}

This report was prepared as an account of work sponsored by an agency of the United States Government. Neither the United States Government nor any agency thereof, nor any of their employees, makes any warranty, express or implied, or assumes any legal liability or responsibility for the accuracy, completeness, or usefulness of any information, apparatus, product, or process disclosed, or represents that its use would not infringe privately owned rights. Reference herein to any specific commercial product, process, or service by trade name, trademark, manufacturer, or otherwise, does not necessarily constitute or imply its endorsement, recommendation, or favoring by the United States Government or any agency thereof. The views and opinions of authors expressed herein do not necessarily state or reflect those of the United States Government or any agency thereof. 


\title{
Beam Transfer at E0: An Overview
}

\author{
S. Saritepe* and G. Annala \\ Fermi National Accelerator Laboratory ${ }^{\dagger}$ \\ Batavia, Illinois, USA
}

June 25,1993

\section{Introduction}

The helical orbits in the Tevatron necessitated changes in the beam transfer operation between the Main Ring and the Tevatron. This document is intended to present an overview of the beam transfer with an emphasis on the recent changes. It will also serve as a bibliography for the other documents that exist on Tevatron injection.

\section{Vertical Plane}

Injection into the Tevatron is accomplished at $150 \mathrm{GeV}$ in the E0 long straight section. The E0 straight section is $150 \mathrm{ft}$ long, the vertical distance between the Main Ring and the Tevatron beam pipes is 25.5 inches thus the vertical bend angle is approximately 17 mrad. Proton transfer is accomplished by four lambertson magnets in the E0 section ( 2 in the Main Ring , 2 in the Tevatron) connected in series and powered by a $500 \mathrm{KW}$ Transrex operating on the $200 \mathrm{~V}, 2500$ Amps tap. There is also a shunt across the Main Ring lambertsons to provide fine adjustment on the bending angles. The arrangement for the antiproton injection line is similar, 2 lambertsons at D49 in the Tevatron and 2 lambertsons at E11 in the Main Ring. Fig.(1) depicts the proton and antiproton injection lines. The shunt for the antiproton injection line is also on the Main Ring (MR) lamberstons. The shunt is not a resistor, it is a transistor bank. It removes current from the E0-MR Lambertsons. Typical currents are 7 Amps on the shunt and 1572 Amps on the main supply. The maximum allowed current on the shunt is 15 Amps. The typical shunt current of 7 Amps corresponds to the nominal MR orbit at E0. Since the MR orbit is subject to frequent tuning procedures, it is often necessary to udjust the shunt current (This is done automatically when one uses the orbit closure program T120). The circuit diagram showing the shunt connections is shown in Fig.(2).

If the vertical MR orbit at E0 is such that a shunt current adjustment of more than \pm 7 Amps is needed, then a local Tevatron orbit bump is performed. The vertical dipole correctors used for this purpose are VD47, VD49 and VE11.

The cross-sectional view of one lambertson is shown in Fig.(3). A schematic section view of the MR and Tevatron lambertson apertures (relative to each other) is shown in Fig.(4).

\footnotetext{
"current address: SSCL, MS 1040, 2550 Beckleymeade Ave. Dallas, TX 75237

${ }^{\dagger}$ Operated by Universities Research Association,Inc., under contract with the United States Department of Energy
} 


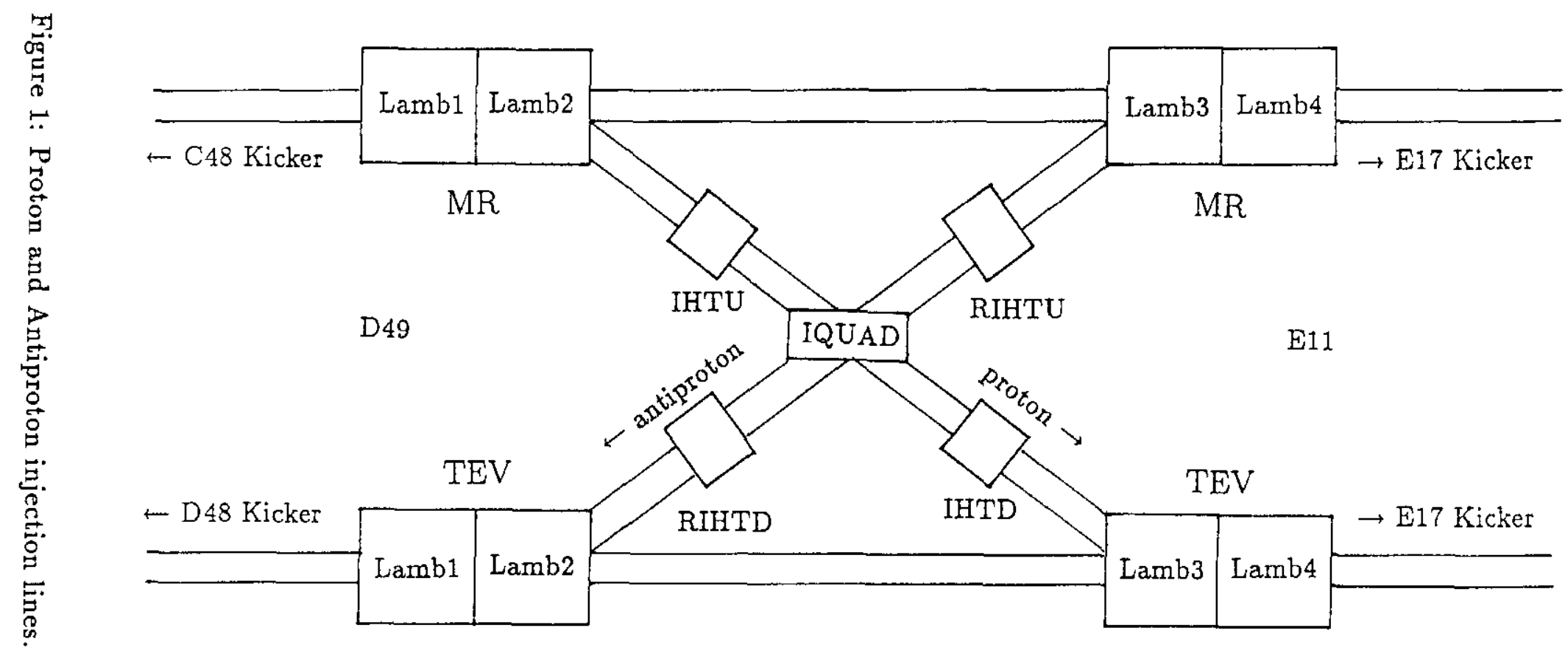




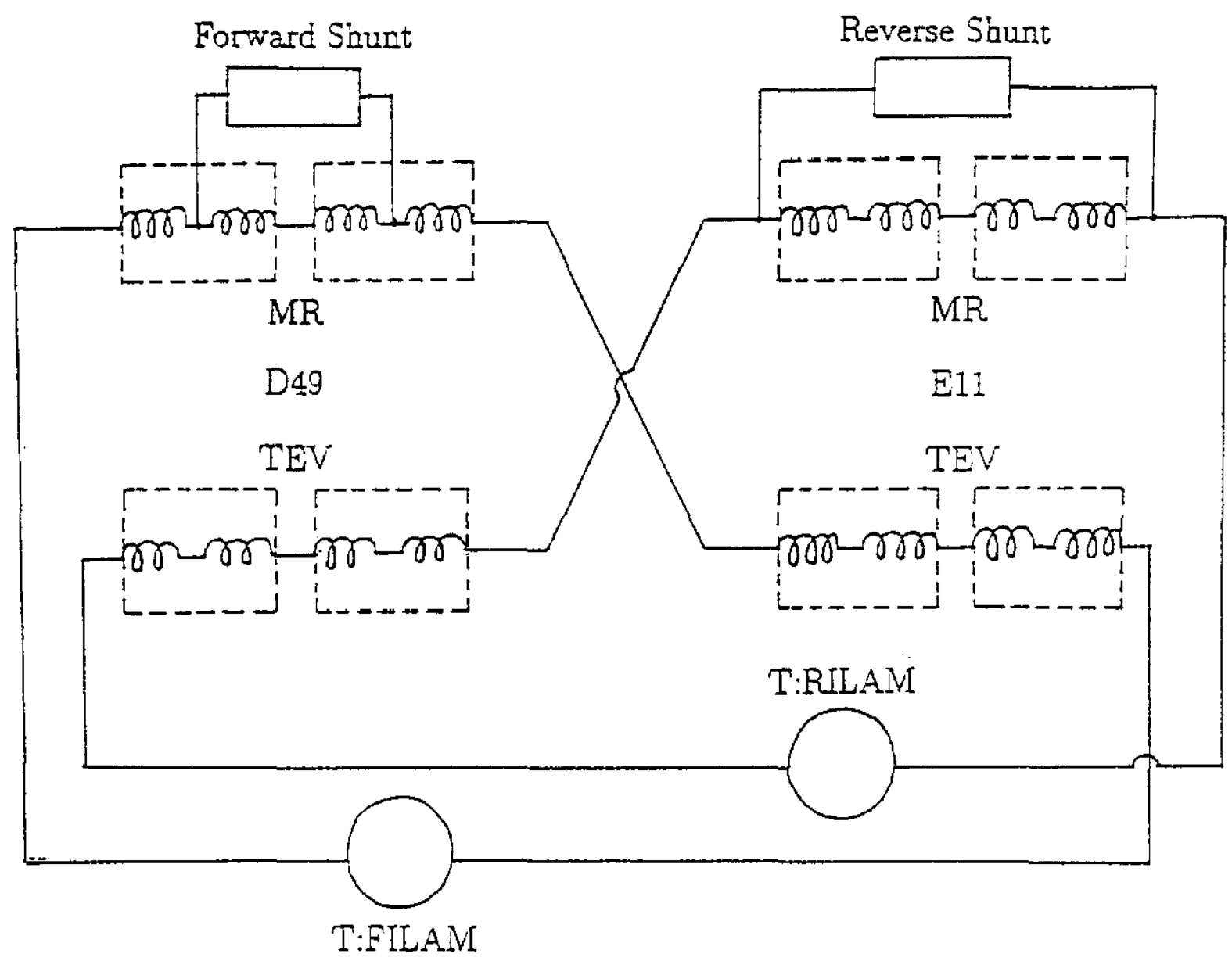

Figure 2: Circuit diagram showing the shunt connections. This diagram was drawn from tunnel observations by G.Annaia. If the forward shunt and the reverse shunt both remove, for instance, 5 Amps from the circuit, the angle adjustment provided by the reverse shunt will be twice as big as that provided by the forward shunt. 


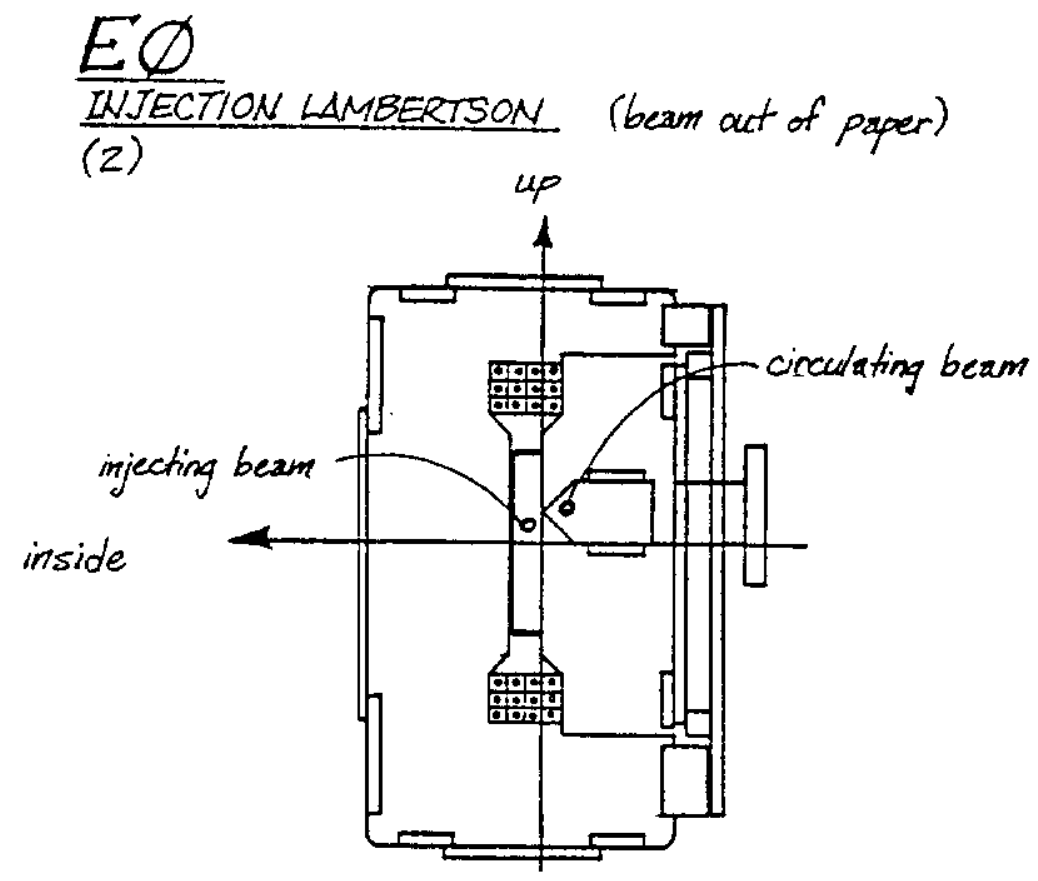

Figure 3: Cross-sectional view of one E0 Injection Lambertson.

Currently, the lambertson currents are controlled by CAMAC 465 cards. The names of interest are:

T:FILAM - forward (proton) injection scale factor

T:RILAM - reverse (antiproton) injection scale factor

$\mathrm{T}$ :FILAMS - forward (proton) injection shunt scale factor

T:ILAMSK - reverse (antiproton) injection shunt scale factor

These are not the standard 465 device names.

\section{Horizontal Plane}

\subsection{Forward (Proton) Injection}

I ) Main Ring

In order to place the proton beam in the field region of the MR lambertsons at D49 a horizontal kick is needed. The $\mathrm{C} 48$ kicker in the Main Ring is used for this purpose. C48 kicker was chosen because it already existed for the fast MR extraction before the Tevatron existed. The C48 Kicker is a $6 \mathrm{~m}$ Ferrite dipole and is powered by the discharge of a "pulse forming network" (PFN) which permits a very fast rise time (360 ns) magnetic field but very large fall-time reflections $(25 \%)$. Fall-time reflections have no effect since the beam has already left the MR when they occur. The nominal setting for the C48 kicker is $52 \mathrm{KV}$ which gives a 0.46 mrad kick. The maximum voltage is $70 \mathrm{KV}$.

The Main ring beam is kicked to the radial inside by the $\mathrm{C} 48$ kicker, after oscillating through the D-Sector, ends up at $\approx 30 \mathrm{~mm}$ to the inside at E0. The horizontal kick of 0.46 $\mathrm{mr}$ causes uncomfortably large beam excursions through the D-Sector. In order to reduce these oscillations 3 horizontal bump magnets were installed in the Main Ring at C22, C32 


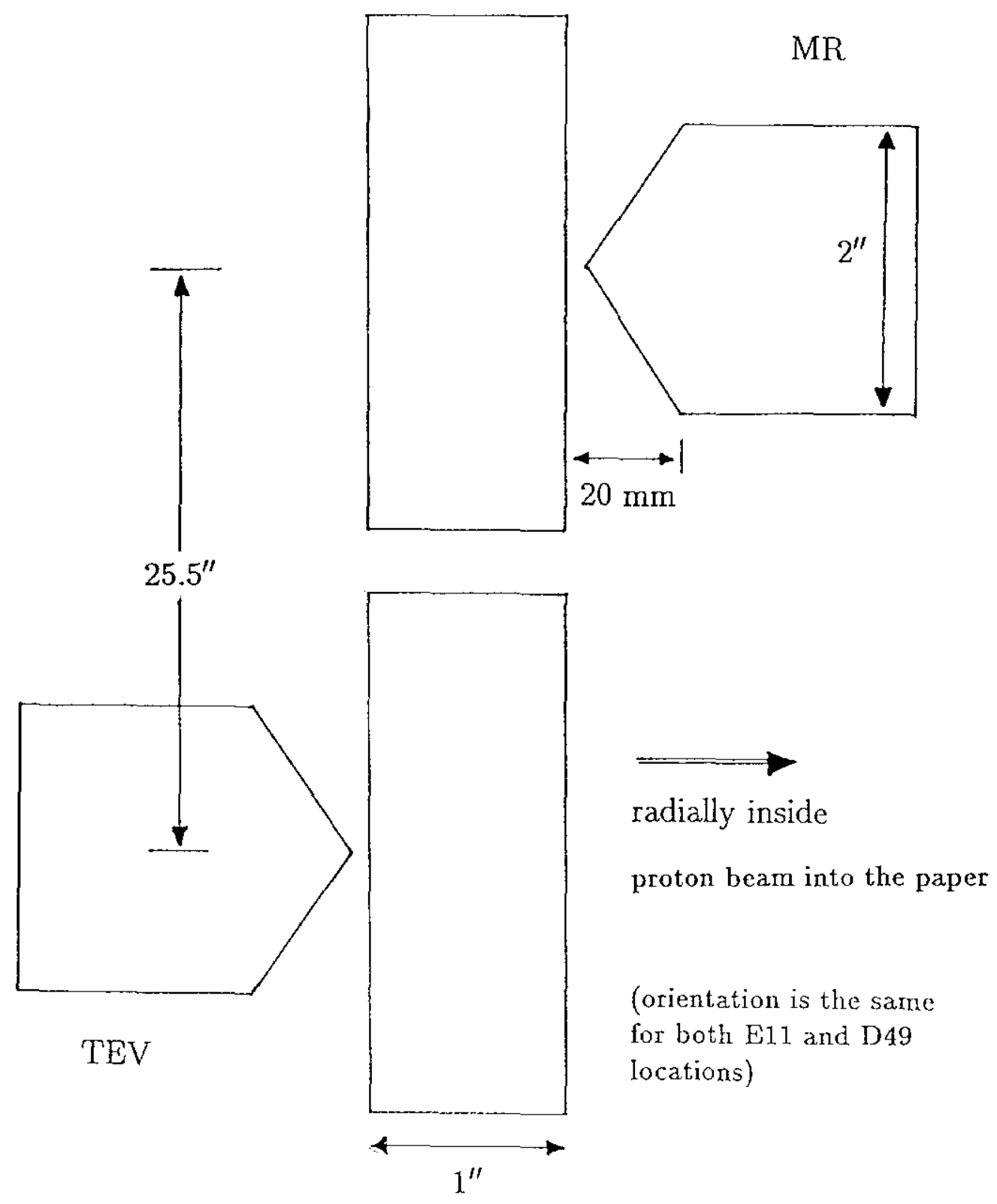

Figure 4: Schematic section view of the MR and Tevatron lambertson apertures. 
and D38 (named T:IBMP1, T:IBMP2 and T:IBMP3, respectively). These are 40 inch iron core dipoles. They form a time bump which produces a closed orbit $180^{\circ}$ out of phase with the kicked beam, thus reducing the large oscillations in the actual orbit.

The precise control of horizontal beam position in the Main Ring at E0 is essential for loss free injection. This is done by another set of bump magnets located at D46 and E17. These are again 40 inch iron core dipoles with separate power supplies.

II ) Forward (proton) injection line

In the forward injection channel there are 3 magnets. In the middle there is a matching quadrupole named IQUAD. The MR and the Tevatron lattices are very similar around E0, the slight difference is matched by this quad. The other 2 magnets are IHTU (upstream) and IHTD (downstream). These are horizontal trim dipoles in the injection line. They are used to adjust the incoming horizontal trajectory into the Tevatron. The control card for IQUAD was recently changed to a CAMAC 465. The relevant device names are

T:FIQUAD - forward (proton) injection scale factor

T:RIQUAI - reverse (antiproton) injection scale factor

\section{III) Tevatron}

Kickers can only be placed in warm sections, which are 17 and 48 locations in the Tevatron. A fast rise time kicker is placed at E17 in the Tevatron to take out the horizontal oscillation initiated by the C48 Kicker in the Main Ring. In contrast to the C48 Kicker, the E17 Kicker is required to have minimal fall time reflections since the beam is circulating in the kicker aperture after the firing of the magnet. The details of this kicker are explained in references [1] and [2]. In summary, a PFN based thyratron switch tube pulser delivers a 100 nsec rise, $200 \mathrm{nsec}$ fall, $40 \mathrm{KV}$ pulse to a $6.25 \Omega$ traveling wave magnet load. The pulse duration is adjustable up to a maximum of $22 \mu \mathrm{s}$. The proton injection line is detailed in Table 1.

\subsection{Reverse (Antiproton) Injection Line}

\section{I ) Main Ring}

In the case of antiproton injection, (T:IBMP1, T:IBMP2 and T:IBMP3) bump in the MR is not needed since the MR beam transfer kicker is placed at E17. Antiprotons perform betatron oscillations in a very short distance between E17 and E11 before they are transfered to the Tevatron. The MR E17 kicker has a half-sine wave form with a rise time of $6 \mu \mathrm{sec}$. The MR E17 kicker will run up to $10 \mathrm{KV}$.

II Reverse (antiproton) injection line

There are 2 trim dipoles for horizontal orbit correction (T:RIHTD, T:RIHTU) and there is the lattice matching quad T:IQUAD. The IQUAD is ramped with the reverse polarity. Tevatron lambertsons at D49 are rolled by $6.5^{\circ}$ (as shown in Fig.(7)).

III) Tevatron

The Tevatron kicker that corrects the injection error is placed at D48. This kicker has 


\begin{tabular}{|c|c|c|}
\hline $\begin{array}{l}\text { Element } \\
\text { (PROTON DIRECTION) } \\
\end{array}$ & ACNET name & $\begin{array}{l}\text { Distance from E0 } \\
\text { (inches) }\end{array}$ \\
\hline Upstream end of Lamb 1 (MR D49) & & -965 \\
\hline Loss Monitor & T:LILM1U & -911 \\
\hline Downstream end of Lamb-1 (MR D49) & & -776 \\
\hline Upstream end of Lamb-2 (MR D49) & & -760 \\
\hline Loss Monitor & T:LILM2U & .578 \\
\hline Downstream end of Lamb-2 (MR D49) & & -571 \\
\hline Horz. BPM & T:PINJUH & -417 \\
\hline Vert. BPM & T:PINJUV & -403 \\
\hline Upstream end of horz. trim & T:IHTU & -375 \\
\hline Loss Monitor & T:LIHTU & -315 \\
\hline Downstream end of horz. trim & $\mathrm{T}: \mathrm{IHTU}$ & -315 \\
\hline Upstream end of the matching quad & T:IQUAD & -17.125 \\
\hline Center of the matching Quad & T:IQUAD & 0 \\
\hline Loss Monitor & T:LIQUAD & +17 \\
\hline Downstream end of the matching quad & T:IQUAD & +17.125 \\
\hline Upstream end of horz. trim & T:IHTD & +315 \\
\hline Downstream end of horz. trim & T:IHTD & +375 \\
\hline Loss Monitor & T:LIHTD & +375 \\
\hline Vert. BPM & T:PINJDV & +403 \\
\hline Horz. BPM & T:PINJDH & +417 \\
\hline Upstream end of Lamb-3 (TEV E11) & & +570 \\
\hline Loss Monitor & T:LILM1D & +570 \\
\hline Loss Monitor & T:LILM2D & +664 \\
\hline Downstream end of Lamb-3 (TEV E11) & & +759 \\
\hline Upstream end of Lamb-4 (TEV E11) & & +775 \\
\hline Downstream end of Lamb-4 (TEV E11) & & +964 \\
\hline Loss Monitor & T:LILM3D & +1000 \\
\hline
\end{tabular}

Table 1: Relative distances in the proton injection channel according to the mechanical drawing "Energy Doubler long Straight E0" (8000-ME-142999 drawn by D.Schmitt). Positions of the loss monitors have been obtained from B.Hanna's notes. 
a rise time of $3 \mu \mathrm{sec}$. The D48 kicker runs at $55 \mathrm{KV}$, very close to its maximum value of $60 \mathrm{KV}$; for this reason it is not used in orbit closure. Currently, there is no orbit closure procedure using antiprotons. The antiproton injection is done after the reverse injection closure which uses the trim dipoles and the MR E17 kicker. The antiproton injection line is detailed in Table 2. The kicker strengths for the Tevatron injection kickers are shown in Table 3.

\section{Operational Issues}

\subsection{Four different orbits at $150 \mathrm{GeV}$}

In the new collider scenario with separated beams and feed-down sextupoles, there are 4 closed orbits at $150 \mathrm{GeV}$. First one is called the "proton injection orbit" which includes a bump at E0. After 6 proton bunches are injected onto this orbit the separators are powered and the proton helix (the second closed orbit) is established. Then 6 antiproton bunches are injected onto the antiproton helix (the third closed orbit). The fourth orbit is known as the "centered orbit". The word "centered" refers to the "orbit smoothing" which was performed during the commissioning phase. In the operational sequence it is a misnomer because it is achieved by subtracting the injection bump while both beams circulate on their respective helices. Protons and antiprotons are accelerated to $900 \mathrm{Gev}$ on their respective "centered" helices.

One switches from the "injection helix" to the "centered helix" and visa versa by adding or subtracting a set of values to corrector dipoles around E0. This bump is currently handled by the 465 cards. The idea of using 465 cards for injection dipoles for a variety of operations was proposed by S. Pruss and implemented by G.Annala [3]. The 3-bumps involved in this switch are: (HD46, HD49, HE11) and (HD49, HE11, HE13) in the horizontal plane; (VD47, VD49, VE11) and (VD49, VE11, VE14) in the vertical plane.

\subsection{Time bumps}

Time bumps are used to create the special orbits which place the beam optimally in the lambertson notch. The main purpose of time bumps is to reduce the kicker strength needed for the beam transfer. Position and angle of the beam relative to the lambertson notch just prior to beam transfer is critically important. Margins are very small. Time bump amplitudes for forward and reverse injection are painstakingly adjusted during commissioning. Once the "operation" settles down on an accepted set of $150 \mathrm{GeV}$ orbits, the time bumps should be left alone. A sketch of the time bump for the proton injection is shown in Fig.(5).

The reverse injection time bump is rather special since it reflects the position and angle required for a loss-free injection of antiprotons onto the helix.

\subsection{Locality of time bumps}

The locality of bumps in general depends on how well we know the lattice. Once we know the lattice parameters the calculation of 3-bumps or 4-bumps is a simple matter. In addition to the uncertainty in our knowledge of the lattice parameters (typically $10 \%$ ) there is also the uncertainty due to ramping rates of the dipoles involved in the time bump. These dipoles are supposed to ramp synchronously. Due to hardware problems they may reach 


\begin{tabular}{|l|l|l|}
\hline \hline $\begin{array}{l}\text { Element } \\
\text { (ANTIPROTON DIRECTION) }\end{array}$ & ACNET name & $\begin{array}{l}\text { Distance from E0 } \\
\text { (inches) }\end{array}$ \\
\hline \hline Loss Monitor & T:LILM3D & +1000 \\
\hline Upstream end of Lamb-4 (MR E11) & & +964 \\
\hline Downstream end of Lamb-4 (MR E11) & & +775 \\
\hline Upstream end of Lamb-3 (MR E11) & & +759 \\
\hline Loss Monitor & T:LILM2D & +664 \\
\hline Loss Monitor & T:LILM1D & +570 \\
\hline Downstream end of Lamb-3 (MR E11) & & +570 \\
\hline Horz. BPM & T:PINJDH & +417 \\
\hline Vert. BPM & T:PINJDV & +403 \\
\hline Upstream end of horz. trim & T:RIHTU & +375 \\
\hline Loss Monitor & T:LIHTU & +375 \\
\hline Downstream end of horz. trim & T:RIHTU & +315 \\
\hline Upstream end of the matching quad & T:IQUAD & +17.125 \\
\hline Loss Monitor & T:LIQUAD & +17 \\
\hline Center of the matching Quad & T:IQUAD & 0 \\
\hline Downstream end of the matching quad & T:IQUAD & -17.125 \\
\hline Upstream end of horz. trim & T:RIHTD & -315 \\
\hline Loss Monitor & T:LIHTD & -315 \\
\hline Downstream end of horz. trim & T:RIHTD & -375 \\
\hline Vert. BPM & T:PINJUV & -403 \\
\hline Horz. BPM & T:PINJUH & -417 \\
\hline Upstream end of Lamb-2 (TEV D49) & & -571 \\
\hline Loss Monitor & T:LILM2U & -578 \\
\hline Downstream end of Lamb-2 (TEV D49) & & -760 \\
\hline Upstream end of Lamb-1 (TEV D49) & & -776 \\
\hline Loss Monitor & T:LILM1U & -911 \\
\hline Downstream end of Lamb-1 (TEV D49) & & -965 \\
\hline & & \\
\hline
\end{tabular}

Table 2: Relative distances in the antiproton injection channel according to the mechanical drawing "Energy Doubler long Straight E0" (8000-ME-142999 drawn by D.Schmitt). Positions of the loss monitors have been obtained from B.Hanna's notes. 


\begin{tabular}{|l|r|r|r|r|}
\hline \hline Device & $\begin{array}{r}\text { Bend } \\
\text { Angle } \\
\text { per Amps } \\
(\mu \mathrm{rad})\end{array}$ & $\begin{array}{r}\text { Max. } \\
\text { Curr. } \\
(\text { Amps })\end{array}$ & $\begin{array}{r}\text { Kick } \\
\text { Angle } \\
\text { per KV } \\
(\mu \mathrm{rad})\end{array}$ & $\begin{array}{r}\text { Max. } \\
\text { Volt. } \\
(\mathrm{KV})\end{array}$ \\
\hline \hline T:FILAM & 10.6 & 2100 & & \\
\hline T:FILAMS & 4.5 & 15 & & \\
\hline T:RILAM & 10.6 & 2100 & & \\
\hline T:ILAMSK & 4.5 & 15 & & \\
\hline T:IHTU & -18.3 & 50 & & \\
\hline T:IHTD & +18.3 & 50 & & \\
\hline T:RIHTD & -18.3 & 200 & & \\
\hline T:RIHTU & +18.3 & 50 & & \\
\hline T:E17K & & & 7.7 & 70 \\
\hline T:C48KIK & & & 8.9 & 70 \\
\hline T:D48K & & & 18.6 & 62 \\
\hline M:E17K3 & & & 131.5 & 10 \\
\hline
\end{tabular}

Table 3: Correction elements. T:IQUAD provides $40 \mu \mathrm{rad}$ kick if the beam misses the quad center by 0.4 inches. Some of the numbers were provided by B.Hanna.

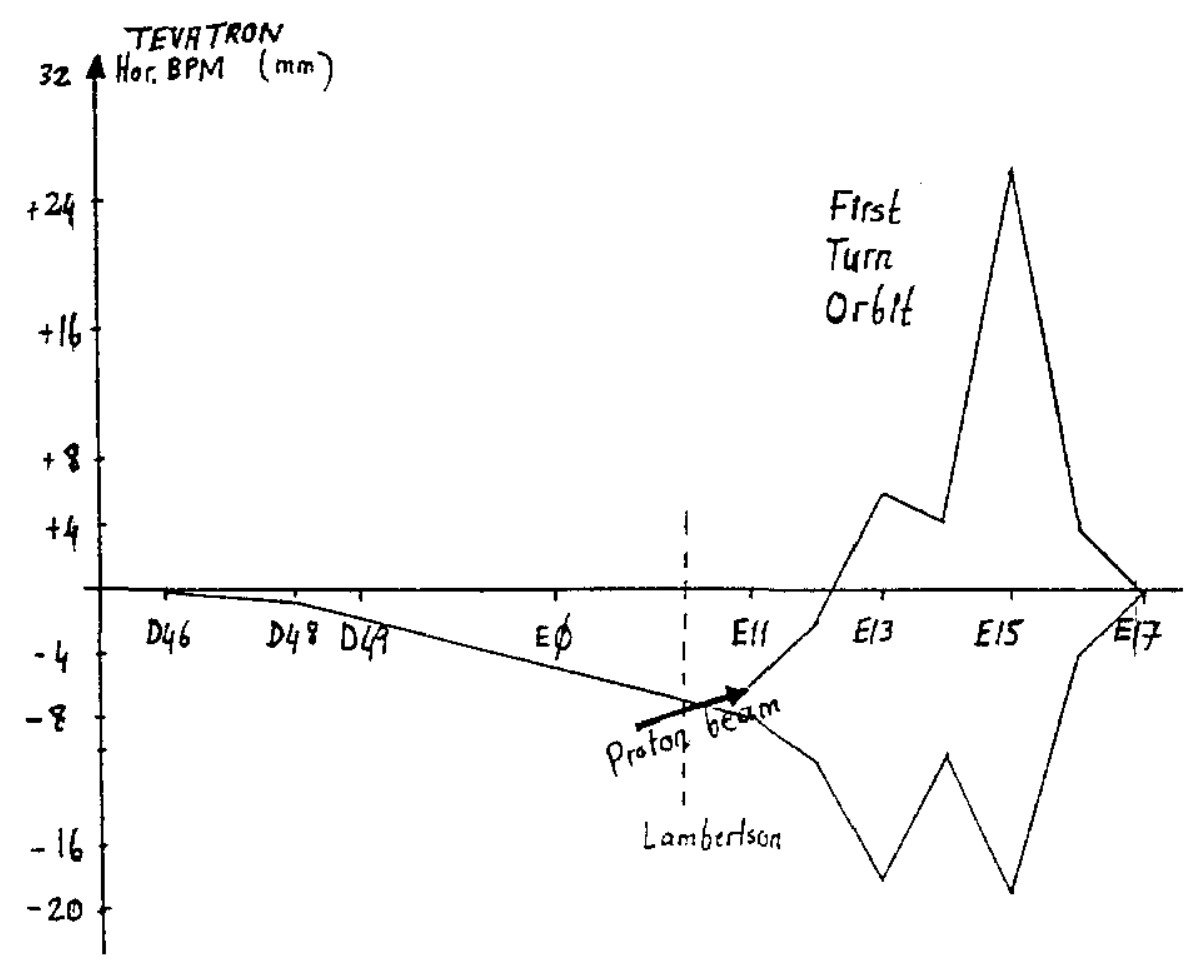

Figure 5: A sketch of the first turn proton orbit and the forward injection time bump. Without the time bump the orbit excursion at E15 would have been more than $40 \mathrm{~mm}$. 

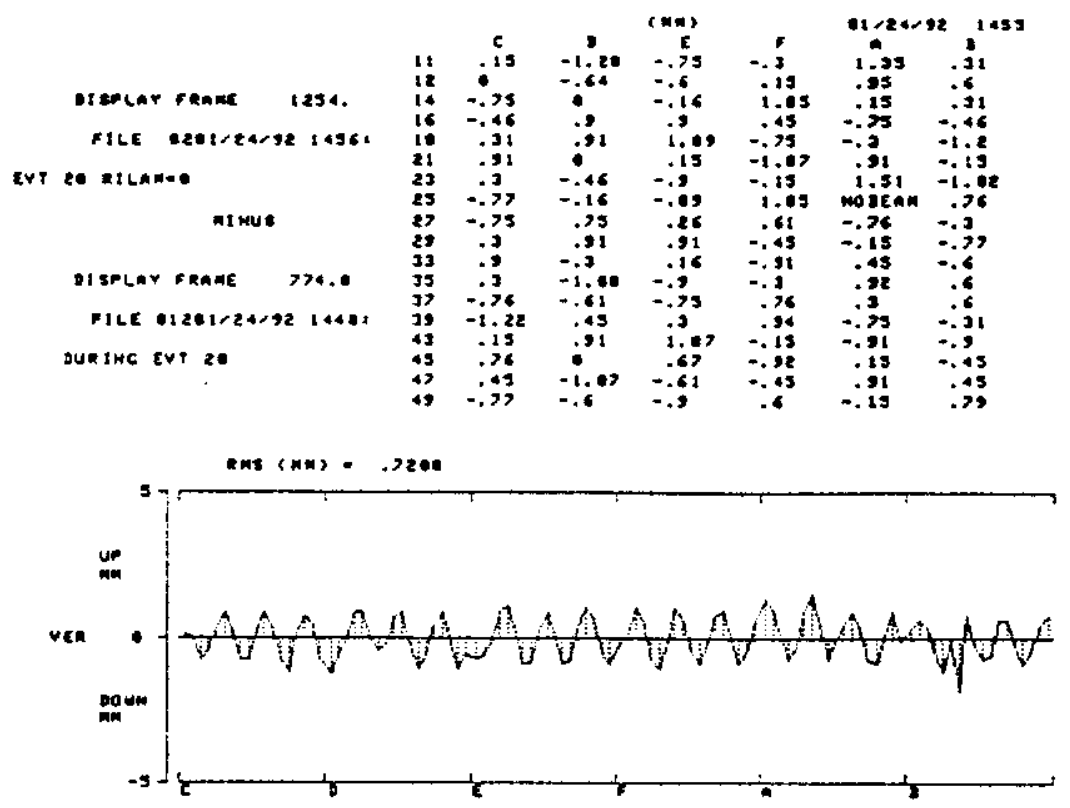

Figure 6: Tevatron orbit distortion caused by the field leakage in the lambertson notch.

their flattop at different times causing the time bump to be nonlocal. It is always a good idea to plot the currents and determine the syncronicity.

\subsection{Comments on field free region of the lambertsons}

The so called "field-free region" of the lambertsons is not really field-free due to the field leakage. The dominant term in the leakage field is the quadrupole term. Therefore there is a tune shift and a closed orbit distortion (feed-down effect) when the lamberstons ramp. This is an issue of course when the lamberstons are in the "injection position" where the notch is relatively close to the beam. Just prior to acceleration and during the acceleration the lamberstons are kept in the "store position" thus making the leakage field effects less important. During the injection cycle, however, there are time-lines with $2 \mathrm{~B}$ or 20 cycles. Forward injection lambertsons ramp on the $2 \mathrm{~B}$ cycles and reverse injection lambertsons ramp on the 20 cycles thus causing the orbit distortions and tune shifts. The vertical orbit distortion caused by the field leakeage is shown in Fig.(6). There are plans to compensate both the tune shift and the orbit distortion effects.

\subsection{New cards for the E0 correction dipoles}

Previously the correction dipoles around E0 were controlled by a combination of CAMAC 160 and 165 cards. The 160 card provided the energy ramp table and the 165 card provided the 2 time tables needed for forward and reverse injection operations. In the new collider scenario there are 4 different $150 \mathrm{Gev}$ orbits. G.Annala implemented the replacement of 165 cards with the 465 cards [3]. In this scheme 160 card contains only an energy ramp corresponding to the "centered orbit" and possibly a time ramp to be played during the low-beta squeeze. The 465 cards contain an energy ramp that represents the difference between the "centered orbit" and the "injection orbit" and 3 time ramps. The first time 
ramp contains the forward injection time bump, the second contains the reverse injection time bump and the third one is used to go from the "injection orbit" to the "centered orbit" at $150 \mathrm{Gev}[3]$.

\subsection{Orbit closure}

During injection into the Tevatron and reverse injection into the MR, there are inevitable errors. The position and angle of the beam at the second kicker may be different than the position and angle exhibited by the closed orbit at that location. The purpose of the closure program is to correct the position and the angle errors for the next injection. For the Tevatron this console application program is T120 [7]. The closure algorithm used by T120 was given by M.Harrison and is very similar to the "Beam Threader" algorithm developed at CERN [8]. The CERN algorithm and the details of the T120 algorithm are described in Appendix A.

\subsection{Do kickers kick radially inside or outside?}

The Tevatron E17 Kicker kicks the beam radially outside [5] whereas the Tevatron D48 Kicker kicks the beam radially inside [5].

\subsection{Lambertson motion}

The Tevatron lambertsons at D49 and E11 can be moved horizontally. When the Tevatron recovers from low-beta, the sequencer moves proton (E11) and antiproton (D49) lambertsons to the so-called "injection" positions. For both type of lambertsons this motion is to the radial outside. The "injection positions" represent the optimal positions that accomodate both the kicker strength and aperture limits.

The forward injection time bump is carefully adjusted so that the protons do not scrape on the antiproton lambertsons during forward injection. The proton lambertsons are moved to the "stored beam positions" (motion towards radial inside) in the "set up p-bar injection" aggregate of the sequencer. In the "prepare to ramp" aggregate which comes after "set up p-bar injection" and "inject p-bars (A1-A6)" aggregates, the antiproton lambertsons are also moved to their "stored beam positions". Only then the switch is made to the "centered orbit". Protons and antiprotons are accelerated to $900 \mathrm{GeV}$ on their respective helices while the tevatron lambertsons at D49 and E11 are in their "stored beam positions".

As we mentioned earlier during lambertson motion the tunes and the orbits change due to the effects of leakage fields in the lambertson notches. Since the console application program C51 is used to move the lambertsons, perhaps the tune compensation should be made within C51 as well [6].

\subsection{Reverse injection}

Antiprotons are too precious to be used for tuning purposes, therefore protons are transfered from the Tevatron back into the Main Ring, tracing the path of antiprotons backwards. This operation is called reverse injection.

In the presence of helical orbits the critical aperture is at the D49 Tevatron lambertsons. In order to accomodate the extra aperture demands the vertical distance between the MR and Tevatron lambertsons at D 49 had to be reduced by $8 \mathrm{~mm}$. The reverse injection time bump and the kicker strength had to changed also. 
Previously, the reverse injection was handled in one supercycle. One batch of uncoalesced bunches was injected into the Tevatron and ejected after 40 seconds. Then the orbit closure was performed in the MR. In the new scheme the lambertson magnets have to be moved, separator polarities have to be switched, activities that cannot be completed in one supercycle. Therefore, the reverse injection sequence was changed. This involved the redefinition of TVBS clock event \$D8 as MRBS \$D8 thus making it possible to inject 6 proton batches (or coalesced bunches) and eject them one at a time on command, performing orbit closure each time in the MR.

These changes, tuning procedures and other reverse injection issues are described in detail in TM-1791 [9]. TM-1791 also contains orbits which can be useful if one has to tune the reverse injection again.

\subsection{Timing issues}

This topic requires a detailed discussion, therefore it is presented in TM-1792 [10]. This document contains tables describing the forward and reverse injection sequences during collider operation and the forward injection during fixed target operation.

\subsection{Aperture measurements}

During the Jan 1992 studies the apertures at D49 and E11 lambertson notches were measured (See Fig.(7) and Fig.(8)). As shown in Fig.(7) there was an aperture restriction for the proton helix. During the shutdown following Jan 1992 studies the Tevatron D49 lambertsons were raised by $8 \mathrm{~mm}$.

\section{Other documents}

In addition to the documents already cited above, there are a few more documents discussing various aspects of the beam transfer between the MR and the Tevatron. There are unpublished notes on beam tuning procedures [11] [12] [13]. These documents contain a wealth of information even though some of the specifics are out of date. Early design features and references to the early design notes can be found in the Tevatron Design Report [14]. A good introduction to Tevatron Injection was written by P.Emma [15]. A design note on the Reverse injection line was written by G.Dugan and M.Harrison [16]. Also by G.Dugan and M.Harrison is a note on kicker and pulser requirements for pbar manipulations [17]. Finally, I would like to refer the readers to TM-1637 written by G.Dugan [18]. This note explains in great detail the bunch spacing options and the beam transfer requirements for Multi-bunch operation of the Tevatron collider. 


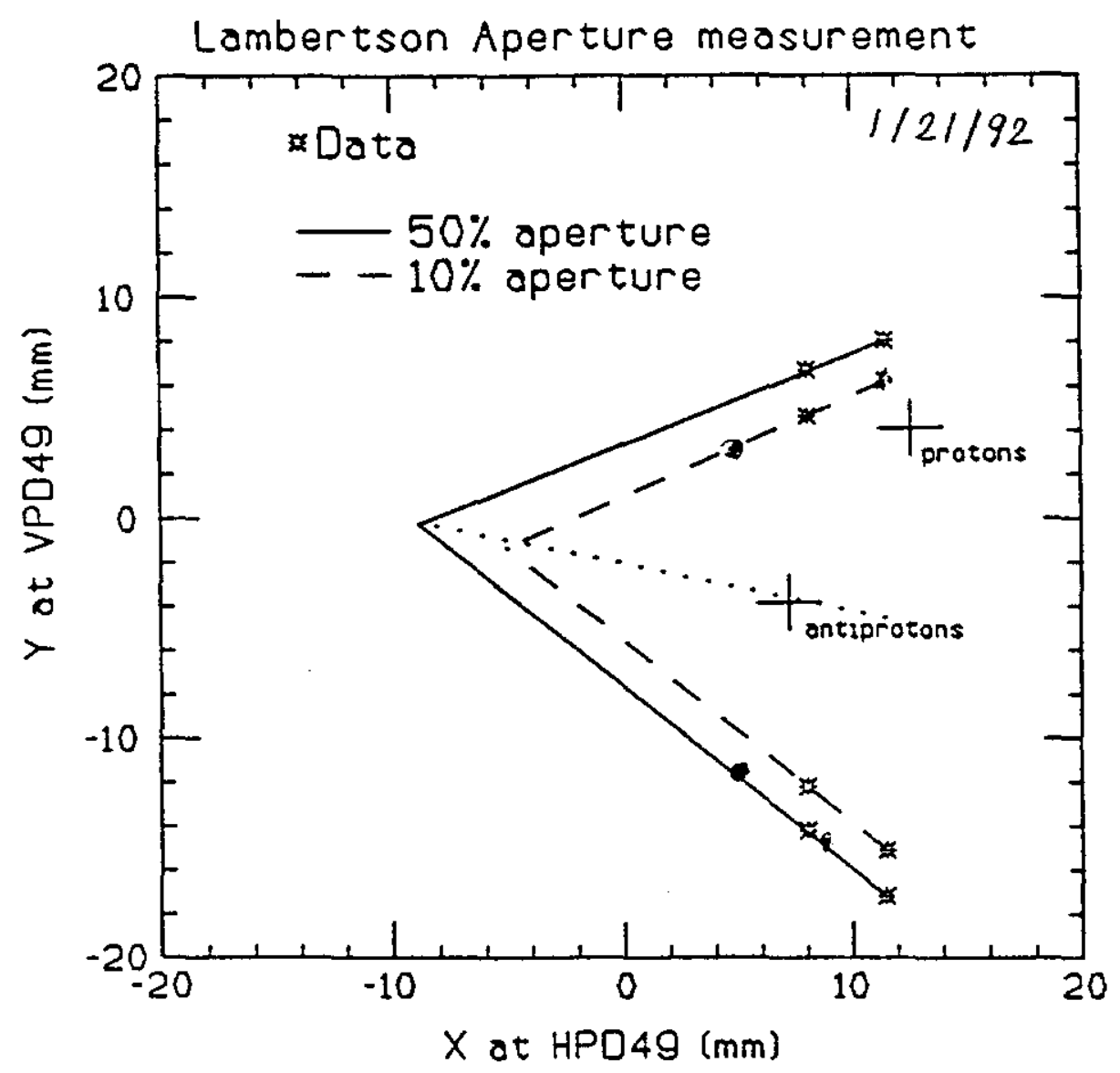

radially outside $\rightarrow$

$\leftarrow$ Lambertson motion towards the "stored beam position"

Figure 7: Measured aperture at the Tevatron D49 lambertsons as of 1/21/92. Later, these lambertsons were raised by $8 \mathrm{~mm}$ providing more aperture for the proton helix. 


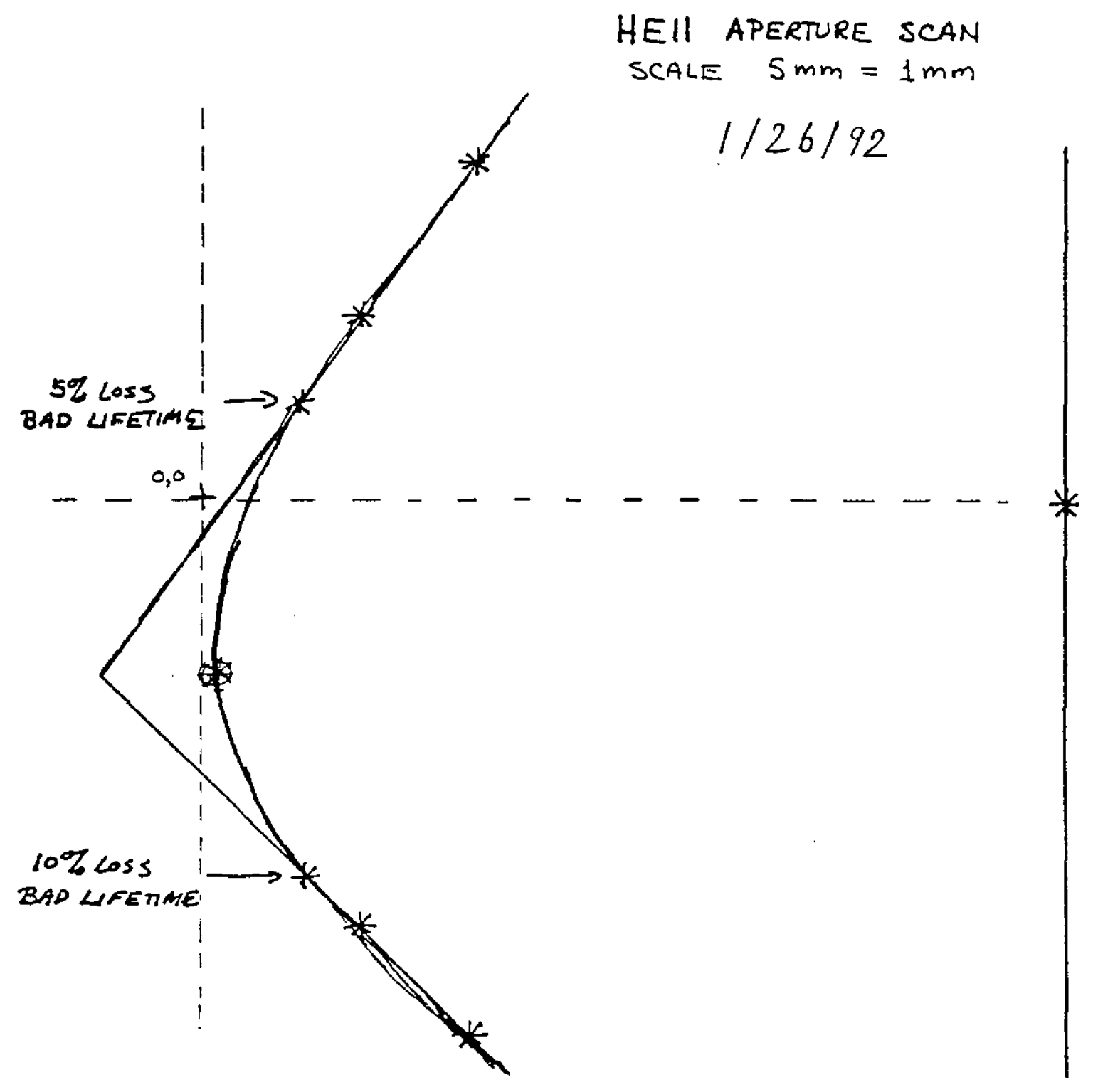

radially outside $\rightarrow$

$\leftarrow$ Lambertson motion towards the "stored beam position"

Figure 8: Measured aperture at the Tevatron E11 lambertsons. 


\section{A T120 and CERN algorithms for orbit closure}

$\underline{\text { The CERN algorithm }}$

1) Subtract the closed orbit from the measured trajectory

2) Define the fitting range (pure oscillation)

3) Read the BPMs in the defined range, modify the numbers according to

$$
\frac{x}{\sqrt{\beta_{x}}}, \quad \frac{y}{\sqrt{\beta_{y}}}
$$

where $\beta_{x}$ and $\beta_{y}$ are the beta values at each BPM.

4) Fit the modified BPM data to

$$
A \sin \left[\psi(s)+\Phi_{0}\right]+\frac{D(s) \delta_{0}}{\sqrt{\beta_{x}}}
$$

where $A$ is the amplitude of the oscillation, $\psi(s)$ is the betatron phase difference between a particular BPM and the first BPM, while $\Phi_{0}$ is the phase difference between the first BPM and the source of the oscillation. $D(s)$ is the dispersion at the BPM locations and $\delta_{0}=\Delta p / p$ is the momentum error at injection. $\Phi_{0}, A$ and $\delta_{0}$ are the unknowns. They are determined from the fit.

5) Select 2 correctors. Flatten the oscillation by exciting the correctors to:

$$
\begin{aligned}
& \theta_{1}=-\frac{A \sin \left(\Phi_{0}-\psi_{2}\right)}{\sqrt{\beta_{1}} \sin \left(\psi_{1}-\psi_{2}\right)} \\
& \theta_{2}=-\frac{A \sin \left(\Phi_{0}-\psi_{1}\right)}{\sqrt{\beta_{2}} \sin \left(\psi_{1}-\psi_{2}\right)}
\end{aligned}
$$

Here, $\psi_{2}$ is the phase difference between the second corrector and the first BPM and $\psi_{1}$ is the phase difference between the first corrector and the first BPM.

\section{T120 Algorithm}

1) T120 looks at the difference between FLASH (first turn) and DISPLAY (closed orbit) data.

2) The fitting range is defined as the following BPMs:

Forward closure: Tevatron BPMs at

Horizontal: HPE19, HPE22, HPE24, HPE26, HPE28, HPE32, HPE34, HPE36, HPE38, HPE42, HPE44, HPE46, HPE48

Vertical: VPE18, VPE21, VPE23, VPE25, VPE27, VPE29, VPE33, VPE35, VPE37, VPE39, VPE43, VPE45, VPE47

Reverse closure: Main Ring BPMs at

Horizontal: HXE19, HXE22, HXE24, HXE26, HXE28, HXE32, HXE34, HXE36, HXE38, HXE42, HXE44, HXE46, HXE48

Vertical: VXE18, VXE21, VXE23, VXE25, VXE27, VXE29, VXE33, VXE35, VXE37, VXE39, VXE43, VXE45, VXE47

3) T120 uses the raw BPM data. It assumes $\beta$ values are the same for all BPMs. 
4) T120 fits the (FLASH - DISPLAY) data to $A \sin \left[\psi(s)+\Phi_{0}\right]$. The constants $\Phi_{0}$ and $A$ are determined from the fit (least-squares minimization). In the CERN algorithm $D(s) \delta_{0}$ is used for the fit but not for sending the corrections. T120 does not use $D(s) \delta_{0}$ in the fit. This neglect would be justified if the dispersion at each BPM were the same, then the effect of $D(s) \delta_{0}$ would be a simple shift. However, dispersion values at different BPMs are different so the $D(s) \delta_{0}$ term should be used in the fit.

5) The following correctors are used by T120:

Forward closure:

Horizontal T:IHTU or T:IHTD (not both) and T:E17K

Vertical T:ILAM and T:FILAMS

Reverse closure:

Horizontal T:RHTD and M:E17K3

Vertical T:ILAMK and T:ILAMSK

\section{References}

[1] M.Harrison, "The Tevatron Injection System", FERMILAB UPC-157.

[2] R.Trendler et al., "The Fermilab Injection Kicker", IEEE Transactions on Nuclear Science, Vol. NS-30, No.4 (1983).

[3] Jerry Annala, "E0 Correction Dipoles", Operations Bulletin 1233.

[4] Jerry Annala, Personal Communication.

[5] Bruce Hanna, Personal Communication.

[6] Joe Longo, Personal Communication.

[7] T120 was written by T.Groves. L.Winterowd made modifications in the sections related to BPM readings. The closure algorithm was specified by M.Harrison.

[8] J.P.Koutchouk, "Trajectory and Closed Orbit Correction", CERN LEP-TH/89-2 (1989).

[9] S.Saritepe and G.Annala, "Tevatron Reverse Injection", FERMILAB TM-1791 (1993).

[10] S.Saritepe and G.Annala, "Tevatron Injection Timing", FERMILAB TM-1792 (1993).

[11] M.Harrison, "Main Ring to TEV Beam Transfer Tuning Procedure" (1988).

M.Harrison, "Reverse Injection Bumps and Kicker Pulse Lengths", Operations Bulletin $1031,(1985)$

[12] Various unpublished notes on Tevatron injection.

[13] Various unpublished notes on Tevatron injection.

[14] Tevatron Design Report.

[15] P.Emma, "Tevatron Injection", Tevatron Rookie Book. 
[16] G.Dugan and M.Harrison, "Dynamics of Pbar Transfer from the Main Ring to the Tevatron", $\bar{p}$ Note 391 (1984).

[17] G.Dugan and M.Harrison, "Kicker and Pulser Systems Required for Pbar Manipulations in the Main Ring and Tevatron", $\bar{p}$ Note 365 (1984).

[18] G.Dugan, "Considerations of Bunch-Spacing Options for Multi-Bunch Operation of the Tevatron Collider", FERMILAB TM-1637, (1989). 